\title{
Use of computer innovative technologies in improving the dartsmen training
}

\author{
Elina Makarova*, Vladislav Dubatovkin, Nataliya Berezinskaya, Lyudmila Barkhatova, Elena Oleynik \\ Russian State Agrarian University - Moscow Timiryazev Agricultural Academy, 127550, Moscow, Russia
}

\begin{abstract}
The research is focused on studying the possibility of effective use of the dart grip system, the work of the athlete's hand, to prepare the dartsman for competitions using the MOSAR complex. The experiment uses optical motion capture systems, a set of video cameras, led parameter sensors, and devices that allow to record the movement of body parts and a dart. This method of training and controlling dart throwing can serve as educational and visual material for training future athletes. The use of such motion capture systems in the near future may become one of the main aspects of training, both beginners and professionals, in many sports.
\end{abstract}

\section{Introduction}

Sport is a multi-faceted activity with a rich set of movements, individual technical skills. It requires a lot of time for training, with a set of necessary effective training methods. Darts is one of the most popular sports games for all ages. Its accessibility and simplicity allows you to try your hand, regardless of the motor experience and health status of the student. Darts has its own technique for performing competitive exercises. It is advisable to analyze the technique of throwing darts by the following elements: preparation, grip, aiming, throw, release, breath control [1]. In the training of dartsmen, an important component is the training of an accurate dart throw at the target. It is important to train the correct stance, the position of the throwing hand and the comfortable holding of the dart that hits the specified sectors of the target. The ability is achieved over years of training [9]. In order to speed up the mastering, modern innovative technologies are used in training activities. MOSAR is an innovative system of 3D technologies and 3D graphics, invented as an analysis tool in the study of movements biomechanics in sports, medicine, and computer animation in the film industry. The MOSAR complex includes optical, magnetic and led parameters that allow you to design a model of a dartsman and determine the most effective technical positions.

\section{Materials and methods}

The study of dartmen's movement techniques was carried out using the MOSAR complex, an innovative 3D technology. The study involved three darts players. They are students of the national team of the University of Russian State Agricultural University named after K. A. Timiryazev.

For this experiment, we used optical motion capture systems, a set of video cameras, and an image processing tool. The accuracy of calculating is determined by the system calibration procedure and depends on the application's tasks. The undoubted advantage of the optical system is the ability to capture movement at very high speed, the absence of restrictions for the athlete when moving, and the great potential for automating the processing process.

For the experiments, 4 high-speed Vicon Vantage cameras with a resolution of 5 MP, a capture speed of up to 2000 frames per second, and $14 \mathrm{~mm}$ markers were used [4]. With each new experience, a different number of reflective markers were attached to the dartsman's body and information about their movement was collected. After that 3D model and skeleton of an animated character were created. The accuracy of them was compared with the real player movement on a five-point scale. Reflective markers were placed in different amounts on the athlete's hand and fingers, depending on their mobility. Three animations were created. They were based on the data obtained from the motion capture system.

\section{Results and discussion}

The purpose of our research is to determine the effective technique for performing a sports exercise in darts. Motion capture systems were studied using a different number of reflectors, projected on a 3D model of a dartsman, with an assessment of the motion transmission accuracy. Demonstration of the motion capture technology usefulness in

\footnotetext{
* Corresponding author: author@email.org
} 
creating a model of movement is necessary to identify technical errors and correct them in the course of training a dartsman.

A grip is a way to hold a dart. The simplest grip technique is used in darts. The main task is to ensure that the dart is securely held and its position is controlled during all phases of the throw. There are several types of grip: three (Fig.2, a), four, and, in rare cases, two fingers [1]. The main task of the aiming throw is to develop the necessary speed of dart's movement in the desired direction and the most rational trajectory to hit exactly at the point of aiming [3].

a

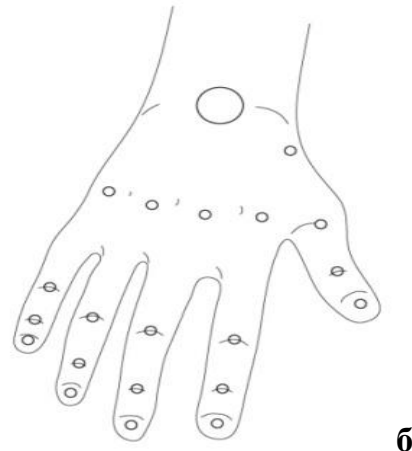

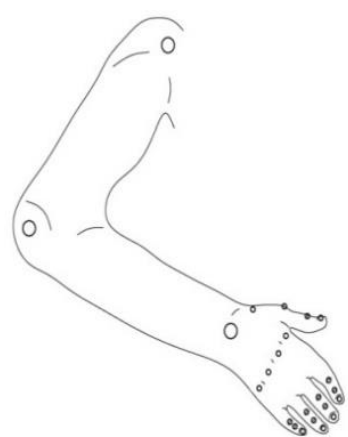

Fig. 1. Markers location on the dartsman's hand and arm.
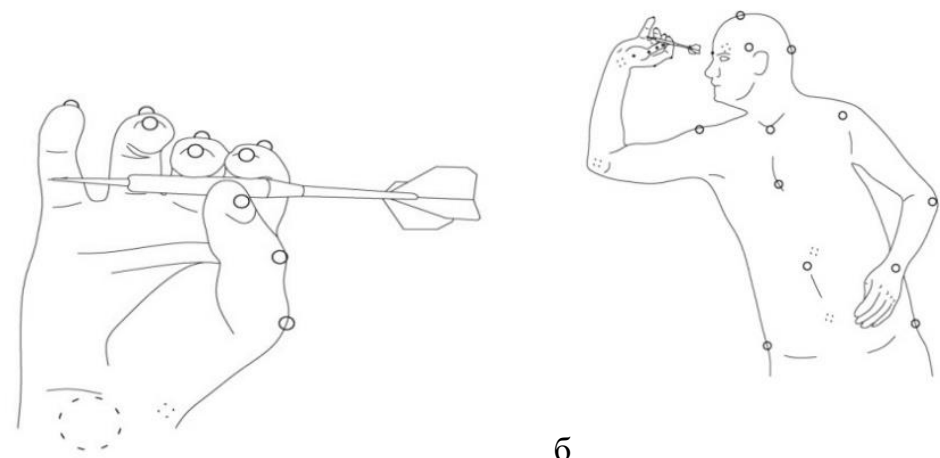

6

Fig. 2. The markers position on the hand during the dart grip and the dartsman's stance.

Reflectors markers were located on the bio-links of the athlete's musculoskeletal system, taking into account the technical features of sports exercises. Figures 1 and 2 show the points of the reflectors: the joints of the fingers, elbow, wrist and shoulder joints of the upper limb, as well as the head and torso of the dartsman. This distribution of markers allows to track the position of the hand, arm and body, fix them on cameras and translate them into a computer projection. Figure 2B shows a dartsman preparing to throw. This is the main stand with a turn angle of 45$60^{\circ}$. The right foot is on the line of the throw. The foot is turned toe forward towards the target at an angle of $45^{\circ}$. The torso is slightly tilted towards the target, the overall center of mass is shifted forward, towards the supporting leg. The angle formed by the throwing arm and the shoulder line is $100-120^{\circ}$. The head is half-turned towards the target and slightly tilted towards the leading hand [2]. This initial position was found in the majority of the tested athletes. It is defined as the most typical for most dart players.

3 leading darts players took part in the pedagogical research. The research was conducted according to the developed scheme:

1) installation of motion capture devices and installation of various number of reflectors on the athlete's body;

2) motion capture is divided into 3 sample models based on data from different numbers of reflectors. Each sample was determined 5 times.

3) creation three $3 \mathrm{D}$ characters in proportion to the athlete's body and attaching the data from the MOSAR system.

Table 1 shows the places and number of markers placed to create an optimal model for controlling the technique of performing a dartsman's sports exercise.

Table 1. Number and location of reflective elements

\begin{tabular}{|l|l|l|l|}
\hline \multirow{2}{*}{ The location of the marker } & \multicolumn{2}{|l|}{ Number of markers placed } & 48 \\
\cline { 2 - 4 } & 41 & 45 & 45 \\
\hline
\end{tabular}




\begin{tabular}{|l|l|l|l|}
\hline Head & 3 & 4 & 5 \\
\hline Shoulders and arms & 23 & 26 & 28 \\
\hline Body & 4 & 4 & 4 \\
\hline Hips & 2 & 2 & 2 \\
\hline Shin & 4 & 4 & 4 \\
\hline Feet & 4 & 4 & 4 \\
\hline Dart & 1 & 1 & 1 \\
\hline
\end{tabular}

Three animations were created. They were based on the data obtained from the motion capture system for three different marker placement options. The models were evaluated by experts on a five-point scale, according to the degree of movements similarity with real players. Based on the results of the estimates, the arithmetic mean and maximum deviation were derived.

Table 2. Quality of the dartsman's throw movement as an animated character

\begin{tabular}{|l|l|l|l|l|l|l|}
\hline \multirow{2}{*}{ Stage } & 41 markers & 45 markers & \multicolumn{2}{l|}{48 markers } \\
\cline { 2 - 7 } & $\bar{x}$ & deviation & $\bar{x}$ & deviation & $\bar{x}$ & deviation \\
\hline Stand & 3,33 & 1,53 & 3,67 & 1,53 & 3,67 & 1,53 \\
\hline Dart grip & 3,00 & 1,00 & 4,33 & 0,87 & 4,67 & 0,58 \\
\hline Aiming & 3,67 & 1,15 & 4,00 & 0,87 & 4,33 & 0,58 \\
\hline $\begin{array}{l}\text { The movement of an arm for } \\
\text { throwing }\end{array}$ & 3,33 & 1,00 & 3,67 & 1,00 & 4,67 & 0,58 \\
\hline Dart throwing & 3,00 & 1,15 & 4,00 & 1,00 & 4,33 & 0,21 \\
\hline $\begin{array}{l}\text { Release of the dart from the } \\
\text { hand }\end{array}$ & 3,33 & 1,15 & 3,33 & 1,00 & 4,33 & 0,58 \\
\hline Dart hit & 3,67 & 0,58 & 3,67 & 1,53 & 3,12 & 1,00 \\
\hline Average & 3,33 & 1,08 & 3,81 & 1,11 & 4,16 & 0,72 \\
\hline
\end{tabular}

Where are the values of $\mathrm{X}$ for the level of correct transmission of traffic:

$4.50 \ldots .5 .00$ - highest accuracy

$3.50 \ldots 4.49$ - high accuracy

$2.50 \ldots .3 .49$-acceptable accuracy

$1.50 \ldots 2.49$ - less accuracy

1.00 to 1.49 - insufficient accuracy[5,6].

From the results of the experiment, it follows that the number and location of reflective markers affects the degree of motion capture accuracy.

The most successful were animations with 48 markers placed on the body of a dartsman. Deviation in the preparation, grip and withdrawal of the hand for throwing a dart, were determined at the same level. The technique of throwing, the speed of the dart, and the accuracy of hitting a given sector vary and are individual in nature[7,8]. The use of a video replay and the MOSAR system clearly revealed typical technical errors. That made it possible to correct them in a timely manner when performing the exercise again. In everyday training, video recording was mainly used, as the use of the MOSAR system in training sessions is quite expensive.

\section{Conclusions}

The novelty of this training method and dart throwing control has sufficient accuracy necessary for the correct transmission of movements to animated 3D models. It can serve as educational and visual material for training future athletes. The use of such motion capture systems in the near future may become one of the main aspects of training, both beginners and professionals in many sports. Therefore, this study determines the novelty and effectiveness of using MOSAR in capturing the movements of professionals and their further transfer to digital models. That helps to create visual modern training materials for athletes and coaches.

\section{References}

1. I.V. Dubatovkin, N.A. Berezinskaya, Darts in the practice of teaching physical education (TVT Divizion, Tutorial - Moscow, 2015)

2. V.I. Dubatovkin, Methods of Training in the Darts. Methodical Recommendations (Publishing House of Russian State Agrarian University-MTAA, M., 2012)

3. A.S. Kuznetsov, Z.M. Kuznetsova, Russian Journal of Physical Education and Sport, 14(4), 5-7 (2019). DOI: 10.14526/20704798-2019-14-4-5-7

4. URL: https://ieeexplore.iee.org/Xplore/home.jsp

5. URL: https://www.researchgate.net/ 
6. V. Bachev, M. Gadev, O. Groshev, P. Yordanov, B. Zlatev, Human. Sport. Medicine, 18(1), 95-102 (2018). DOI: $10.14529 / \mathrm{hsm} 180108$ https://elibrary.ru/item.asp?id=32676828

7. V.V. Khramov, E.V. Shirshova, E.L. Matova, Human. Sport. Medicine, 19(S1), 99-105 (2019). DOI: 10.14529/hsm19s113 https://elibrary.ru/item.asp?id=39146805

8. A. Tsos, V. Pasko, A. Rovniy, O. Nesen, I. Pomeshchikova, V. Mukha, Physical Activity Review, 6, 257-265 (2018). doi: 10.16926/par.2018.06.30https://elibrary.ru/item.asp?id=38671156

9. A. Rovniy, V. Shutieiev, V. Ashanin, V. Pasko, Journal of Physical Education and Sport, 19(1), 1368-1373 (2019) 